\title{
SAR Simulation of Ocean Scenes Covered by Oil Slicks with Arbitrary Shapes
}

\author{
Alessandro Danisi, Gerardo Di Martino, Antonio \\ Iodice, Daniele Riccio, Giuseppe Ruello \\ Department of Electronic and Telecommunication \\ Engineering \\ Università di Napoli "Federico II" \\ Napoli, Italy. \\ \{gedima, iodice, riccio, ruello\}@unina.it
}

\author{
Marivì Tello, Jordi J. Mallorqui, Carlos Lopez- \\ Martinez \\ Department of Signal Theory and Communications \\ Universitat Politècnica de Catalunya \\ Barcelona, Spain \\ \{marivi.tello, mallorqui, carlos.lopez\}@tsc.upc.es
}

\begin{abstract}
The identification of oil slicks on the ocean surface from SAR data requires quantitative sound models accounting for the most important characteristics (ocean spectrum, slick viscosity, slick shape, and so on). In this paper we present the implementation of an innovative SAR raw signal and image simulator, which is able to reproduce images relative to ocean surfaces covered by oil slicks with arbitrary shapes. The attention is mainly focused on slicks with fractal contours. The fractal Weierstrass-Mandelbrot function is used to generate slicks with fixed fractal dimension. A box counting technique is employed to evaluate the fractal dimensions of the generated slicks and the corresponding SAR images. Radiometric properties of the area covered by oil are also estimated in order to show how the simulated data provide a powerful set for processing algorithms.
\end{abstract}

Keywords: Synthetic Aperture Radar; oil slick; pollution detection.

\section{INTRODUCTION}

It is widely known that oil slicks on the ocean surface can be observed in Synthetic Aperture Radar (SAR) images as dark spots [1]-[3]. Nevertheless, the use of SAR data is still limited because dark areas in SAR images can be due to several phenomena, as lack of wind, natural oil, plankton, and so on. So far, this ambiguity limited the development of automatic oil detection procedure.

The comprehension of the SAR images can be strongly improved if reliable direct models, accounting for the dominant physical phenomena (wind history, slick nature, sensor characteristics, and so on) are available. Recently, a SAR raw signal simulator of ocean scenes covered by oil slicks was presented [2]. Such a simulator relies on efficient models of the ocean description and of the electromagnetic-ocean wave interaction. The ocean surface is described by means of a two scale model, including long (periods of about 100 meters), and short (periods of $\mathrm{cm}$ and $\mathrm{mm}$ ) waves. In addition, intermediate scale waves are accounted for as an effect on the signal. A model based on the Marangoni theory accounts for the oil slick effect as well [2]. The main limit of this simulator is that it was not possible to define a shape for the slicks.
In order to enlarge the range of applications of our simulator, in this paper we extended the simulator potentiality, providing the chance of generating slicks with arbitrary shapes. In particular, we focused our attention on the simulation of oil slicks with fractal shapes, because it is widely accepted that the fractal geometry is the most suitable instrument to generate natural profiles. In particular, we used the Weierstrass Mandelbrot (WM) fractal function to synthesize a fractal surface. Such a model is a superposition of sinusoidal tones spaced by an irrational parameter, hence it is an almost periodic function [4]. In Section II we present the basic concepts on which the generation of the oil slick shape was addressed.

Once the oil slick was generated, we employed the obtained results as input for the SAR raw signal simulator. In particular, we simulated SAR images relative to fractal slicks as well as slicks with integer dimension, in order to emulate the characteristics of a ship emission. In Section III the basic concepts of the SAR simulation are recalled and the obtained images are shown.

One of the techniques often used to discriminate between natural and man-due slicks is the regularity of the contour. Therefore, in Section IV, we analyzed the obtained images, with the double goal of verifying the law of conservation of the fractal dimension, as well as the radiometric behaviour of the oil covered area with respect to clean water. In Section V we draw the final conclusions, and we underline the activities opened by the new potentialities of the presented simulator.

\section{Generation Of The SLick Shape}

The fractal geometry is widely recognized as the most appropriate instrument for the description of natural shapes. The estimation of the fractal dimension can be a mean for discriminating between natural and man-due slicks. Therefore, it can be interesting for oil detection purposes, to generate slicks with fractal shapes. In order to do it, the most appropriate function is the fractional Brownian motion ( $\mathrm{fBm})$, which is a process described in terms of the pdf of its increments [5]. In this paper we synthesized a realization of a $\mathrm{fBm}$ process by means of the Weiersrass-Mandelbrot function, which is a 
superposition of sinusoidal tones, spaced by means of an irrational parameter $v$.

$$
z(x, y)=A \sum_{p=0}^{M-1} C_{p} v^{-H \cdot p} \sin \left[k_{0} v^{p}\left(x \cos \Psi_{p}+y \sin \Psi_{p}\right)+\Phi_{p}\right] \text { ( }
$$

In (1) $A$ is the vertical overall height scaling factor, $C p, \Phi_{p}$, $\Psi p$, are random amplitude, phase and direction coefficients, $H$ the Hurst coefficient $(0<H<1)$, related to the fractal dimension $D_{W M}$ by means of the relation $D_{W M}=3-H, k_{0}$ the fundamental wavenumber.

A cut of the WM function at fixed $\mathrm{z}$ levels provides a fractal curve which defines the region covered by the oil. As an example, in Fig. 1 we depict a slick obtained by a cut of a WM function with fractal dimension $D_{W M}=2.35$. The image was synthesized on a $3.99 \times 19.9 \mathrm{~m}^{2}$ spaced grid, in accordance with the ERS-1 acquisition geometry. The image pixel of Fig. 1 has a $19.9 \mathrm{~m} \times 19.9 \mathrm{~m}$ (azimuth $\mathrm{x}$ range) dimension, in order to show the ground aspect of the slick. Therefore, such an image can be used as an input for the simulation of ERS-1 raw signals and images.

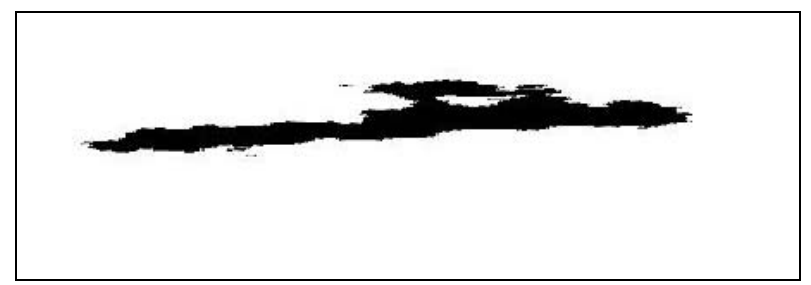

Figure 1. A fractal slick obtained as a cut of a WM function with fractal dimension $D_{W M}=2.35$.

In order to estimate the fractal dimension of the slick contour, we applied a box counting technique, and we obtained a fractal dimension $D c=1.38$. It means that difference between the Hurst coefficient $H$ of the contour (related to the fractal dimension $D_{c}$ by the relation $D_{c}=2-H$ ) and that of the corresponding WM process is less than $4 \%$. Similar results were found for different slicks.

In several cases, it is of interest to discriminate between natural and man-due slicks, by evaluating the regularity of the contour. Therefore, we generated slicks whose shapes recall the typical slicks spilled by the boats, by using the classical geometry. As an example, in Fig. 2 we present the shape of a slick, obtained as a brunch of a spiral function.

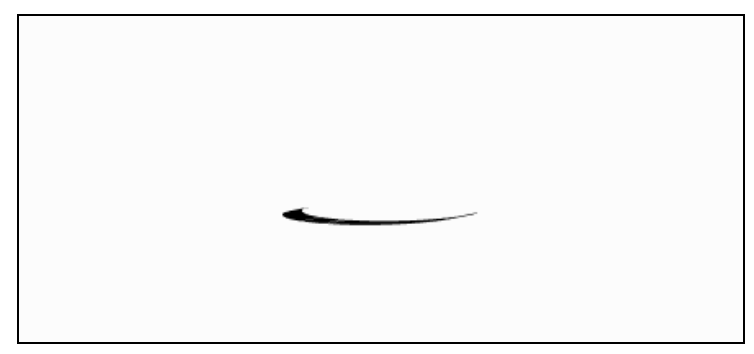

Figure 2. A slick obtained as a branch of a spiral.

\section{SAR SimULATION OF OCEAN SCENES}

In this section we recall the most important concepts for the SAR raw signal simulation of oil slicks on the ocean surface and we show the simulations relative to oil slicks with arbitrary shapes.

Let $t$ be the independent time variable, $x$ and $r$ the independent space variables, standing respectively for azimuth and range. By using prime coordinates for the SAR raw signal, this can be expressed as [2]:

$$
s\left(x^{\prime}, r^{\prime}\right)=\iint d x d r \gamma\left(x, r, x^{\prime}=v_{s} t\right) g\left(x^{\prime}-x, r^{\prime}-r ; r\right),
$$

where $\gamma\left(x, r, x^{\prime}\right)$ is the (time-varying) reflectivity pattern of the ocean, $v_{s}$ the sensor velocity, and $g\left(x^{\prime}-x, r^{\prime}-r ; r\right)$ the unit impulse response of the SAR system.

The evaluation of the reflectivity function requires a description of the ocean scene and a model of the interaction between electromagnetic and ocean waves. Here the ocean surface is described as a superposition of a swell and a roughness of the order of the incident wavelength. In addition, the area covered by the oil is defined according to the concepts described in Section 2. In addition, the time variant nature of the ocean, in our simulator, is included via the Harger DS model. According to this model, the time dependence can appropriately "migrate" from the reflectivity function to the impulsive response function provided that a dispersion relation for the long wave spectrum is available [2].

As for the electromagnetic model, in ocean environment, the SAR signal intensity turns out to be proportional to the ocean spectrum at spatial frequencies given by the Bragg theory. The oil influence is accounted for via the Marangoni theory [2], as a damping of the ocean spectrum. The intensity of the damping depends on the oil properties and it is presents a resonant behaviour. Therefore, the best sensor for oil detection depends also by the oil resonant frequency. As an example, the Oleyl Alchool, which is here considered as case study, presents a resonance around spatial frequency that correspond to the $\mathrm{C}$ band [1], [8]. The SAR unit impulse response is evaluated once the sensor parameters are defined.

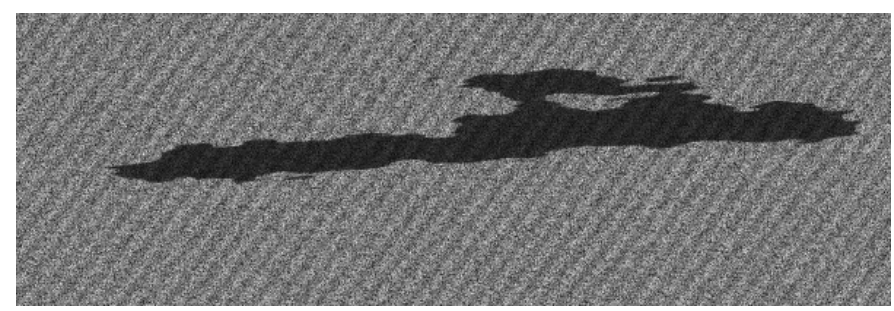

Figure 3. Simulated ERS-1 SAR image relative to the slick synthesized in Fig. 1. The near range is on the left.

The C-band ERS-1 SAR image relevant to the oil slick whose shape was shown in Fig. 1 is obtained from the simulated raw signal (1) via standard processing and presented in Fig.3. The most relevant sensor parameters are provided in the first column of Table I. The image is averaged with a $1 \times 4$ multi-look, so that its resolution in azimuth - slant range is 
$19.9 \times 7.9 \mathrm{~m}^{2}$, which corresponds to an azimuth - ground range approximately square pixel $\left(19.9 \times 19.9 \mathrm{~m}^{2}\right)$.

TABLE I. SAR SENSOR PARAMETERS

\begin{tabular}{|l|c|c|}
\hline \multicolumn{1}{|c|}{ Sensor parameters } & ERS-1 & SIR-C \\
\hline Platform Height [Km] & 775.8 & 225 \\
\hline Look Angle [deg] & 23.3 & 46.7 \\
\hline Carrier Frequency [GHz] & 5.3 & 1.25 \\
\hline Chirp Bandwidth [MHz] & 15.5 & 10 \\
\hline Field Polarization & HH & HH \\
\hline
\end{tabular}

In order to show the image dependence on the carrier frequency we simulated the SAR image signal relative to the previous scene, acquired by the SIR-C sensor, at L band. The most relevant sensor parameters are presented in the second column of Table I. In Fig. 4 the corresponding SAR image is shown. The image is averaged with a $1 \times 4$ multi-look, so that its resolution in azimuth - slant range is $18.3 \times 13.3 \mathrm{~m}^{2}$, which corresponds to an azimuth - ground range approximately square pixel $\left(17.7 \times 18.3 \mathrm{~m}^{2}\right)$. As expected, the image damping is strongly reduced, because the working frequency is in an area where the spectral damping is lower.

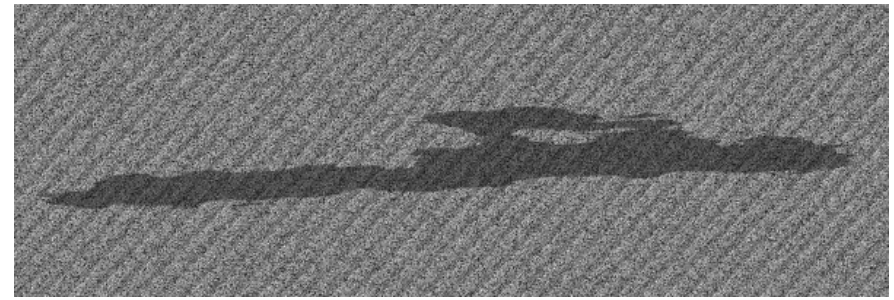

Figure 4. Simulated SIR-C SAR image relative to the slick synthesized in Fig. 1. The near range is on the left.

In the following, we present also the SAR simulation relevant to a slick with the classical contour presented in Fig. 2. The slick properties were chosen with the goal of reproducing the geometrical characteristics of a slick spilled by a boat.

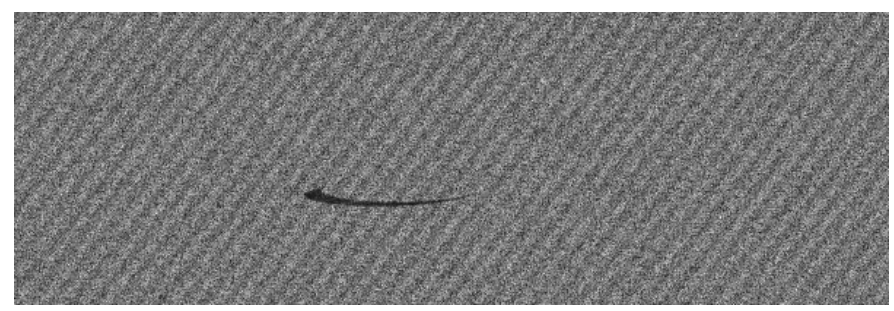

Figure 5. Simulated SAR image reproducing a boat emission. The near range is on the left.

The introduced ability of the simulator can also be exploited to account for a typical ambiguity problem, related with the lack of wind. In fact, it is now possible to generate arbitrary shaped regions where the wind intensity is supposed to be reduced with respect to the background. As an example, in Fig. 6 we show a simulated ERS-1 image with the contemporary presence of an oil slick and a lack of wind.

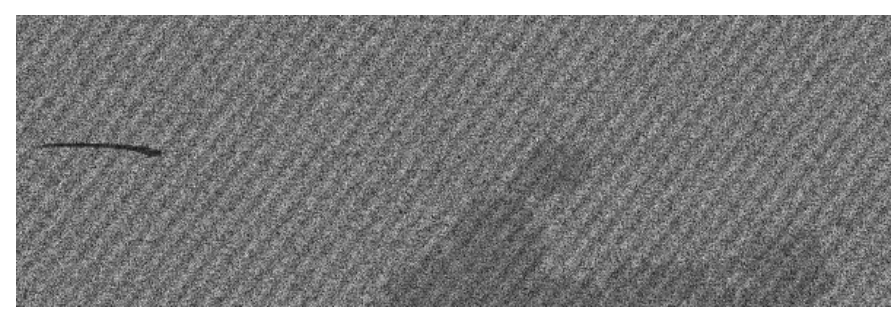

Figure 6. Simulated SAR image relative to a boat emission (on the left ), and a lack of wind (on right). The near range is on the left.

\section{ANALysis Of the Simulated IMAges}

The presented simulator can be intensively exploited to generate a set of images that can be used to improve the comprehension of the image characteristics and for training of detection techniques. In addition, the ability of simulating lack of wind and oil slicks gives the opportunity of facing the problem of the ambiguity solution as well. In the following, we present some examples on how simulated images can be a support for developing SAR techniques. In particular we focus our attention on the estimation of the slick contour fractal dimension and the analysis of the slick radiometric properties.

\section{A. Fractal dimension estimation}

It is widely known that the natural shapes present fractal behavior. Therefore, in order to discriminate between natural and man-driven slicks, the estimation of the slick contour fractal dimension can be a classification criterion.

As an example, we estimated the fractal dimension of the SAR images relative to the slicks generated in Section II. The evaluation of the fractal dimension is possible if the pixel belonging to the slick are identified. This operation can be performed by defining a threshold image intensity value. Of course such an approach is limited by the speckle noise that makes ambiguous the pixel classification.

Anyway, the combined use of morphological operators allows the extraction of the slick contour. As an example, in Fig. 7 we show the contour extracted by the SAR image presented in Fig. 4.

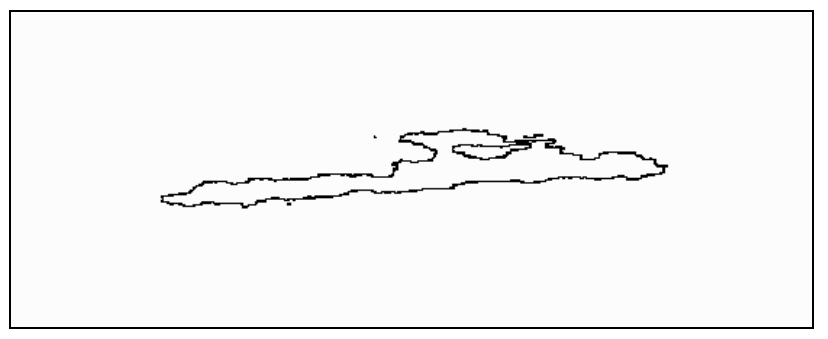

Figure 7. Contour extracted by the SAR image of Fig. 4. 
The contour fractal dimension was then evaluated via a box counting algorithm, obtaining a value $D_{c}=1.3$. As expected, the fractal dimension is similar to the slick mask, slightly reduced by the morphological operations required to limit the speckle noise effect. Similar results were obtained for fractal slicks with different fractal dimension.

The evaluation of the fractal dimension was also performed on the image presented in Fig. 5. Note that the extension of the slick is much more limited, therefore, the extraction of the contour and the evaluation of the fractal dimension can suffer for reliability problems. The obtained fractal dimension value $D_{c}=0.9$ confirms that the slick contour does not hold fractal property. It appears clear that the evaluation of the slick contour fractal dimension can be a technique for ambiguity removal.

\section{B. Radiometric techniques}

The slick classification is often addressed via the combined use of the contour fractal dimension retrieving and techniques based on the analysis of the SAR image radiometric properties. The presented simulator provides a huge amount of data for the developing and training of radiometric techniques as well. In the following, we recall an innovative technique for the discrimination between oil slicks and lack of wind, argued by theoretical considerations, and verified with the use of simulated data [2].

As stated in section III, the intensity of the electromagnetic return scattered by a portion of the sea surface is proportional to the ocean spectrum at frequencies sampled by the Bragg relation. The oil presence damps the ocean spectrum and it changes the higher order statistics, at variance of a lack of wind, which causes a rigid damping of the ocean spectrum.

Therefore, the estimation of the normalized intensity moments on several simulated SAR images led to verify that the higher order moments in areas covered by oil are modified with respect to the clean water and to areas where a lack of wind is present [2]. The main limit of this technique was related to the fact that in actual cases the slick dimension often does not provide a sufficient population for evaluating reliable statistics. Anyway, the extension of the simulator presented in this paper allows the generation of a huge set of SAR images, devoted to quantitatively face the study of the limits of the technique, in terms of the slick geometrical characteristics.

\section{CONClusions ANd Future WORK}

In this paper we presented an innovative simulator, able to synthesize SAR images of oil slicks with arbitrary shapes. The simulator is based on sound electromagnetic and oceanographic models. We focused our attention on the generation of fractal (accounting for natural slicks) and classical shapes (accounting for boat emissions).

The presented simulator permits to synthesize slicks with assigned fractal dimension by using an appropriate cut of WM processes. The Hurst coefficients of the slick contour and of the WM process turn out to be very close (a maximum difference of $4 \%$ was noticed). The fractal dimension of the corresponding SAR image turns out to be slightly reduced, probably by the processing required to extract the slick contour.

The obtained results appear to be very attractive for answering to still open questions. In fact, the intensive use of the simulator allows to generate a complete set of SAR images, whose analysis can lead to shed light on the relationship between fractal dimension of the slick and of its SAR image; the minimum SAR image dimension for obtaining reliable results; the effect of the speckle on the information retrieving process; the minimum damping that allows the extraction of contour information, and much more.

\section{REFERENCES}

[1] W. Alpers and H. Huhnerfuss, "The Damping of Ocean Waves by Surface Films: A New Look at an Old Problem”, J. Geophys. Res., 94, 6251-6265, 1989.

[2] G. Franceschetti, A. Iodice, D. Riccio, G. Ruello, R. Siviero, "SAR Raw Signal Simulation of Oil Slicks in Ocean Environment", IEEE Trans. Geosci. Remote Sens.,vol.40, n.9, pp. 1935-1949, September 2002.

[3] J. C. Scott, "Ocean Surface Slicks - Pollution, Productivity, Climate and Life Saving", Proc. IGARSS '99, Hamburg, 1999.

[4] M. V. Berry and Z.V.Lewis, "On the Weierstrass-Mandelbrot Fractal Function", Proc. R. Soc. Lond. A, 370, 459-484, 1980.

[5] B. B. Mandelbrot and J. W. Van Ness, "Fractional Brownian Motions, Fractional Noises and Applications", SIAM Review, vol.10, pp. 422-437, October 1968.

[6] M. Gade, W. Alpers, H. Huhnerfuss, H. Masuko and T. Kobauashi, "Imaging of Biogenic and Anthropogenic Ocean Surface Films by the Multifrequency/Multipolarization SIR-C/X-SAR", J. Geophys. Res., $18,851-866,1988$ 